\title{
The novel diagnostic marker in low-LVEF heart failure patients
}

\author{
Demir $\mathrm{S}^{1}$, Ede $\mathrm{H}^{2}$, Kaplan $\mathrm{M}^{1}$, Yavuz $\mathrm{F}^{1}$, Yucel $\mathrm{C}^{1}$, Kurt $\mathrm{IH}^{1}$ \\ Adana City Hospital, Health and Science University, Cardiology Department, Adana, Turkey. \\ kardiomehmet27@hotmail.com
}

\begin{abstract}
OBJECTIVE: The structural and compositional changes in the myocardium seem to have a major role in the development of heart failure (HF).Imbalance between production and degradation in extracellular collagen results in increase of collagen synthesis biomarkers in the circulation as the carboxy-terminal propeptide of type I procollagen (PIP). Here we aimed to determine role of PIP in the diagnosis of chronic HF.

MATERIAL AND METHODS: 87 patients with HF group and 80 healthy subjects were enrolled into the study. Echocardiographic examination was performed.At the beginning of the study, serum $B$ type natriuretic peptide (BNP), PIP, high sensitive C-reactive protein (hs-CRP) were measured. The subjects were followed for one year then after.

RESULTS:Average PIP value of HF group was significantly higher than that of the control group ( $p<0.001$ ). Both hs-CRP and BNP values were well correlated to PIP values $(p<0.001)$. In the HF group, PIP value of patients who died at the end of one year was similar to that of patients who survived at the end of first year. CONCLUSION: PIP may not mirror acute events in follow-up of chronic heart failure but it is a very beneficial biomarker in diagnosis of low-LVEF heart failure with high sensitivity and specificity (Tab. 2, Fig. 1, Ref. 16). Text in PDF www.elis.sk.

KEY WORDS: PIP, BNP, hs-CRP.
\end{abstract}

\section{Introduction}

The structural and compositional changes in cardiomyocyte and noncardiomyocyte compartments of the myocardium seem to have a major role in the development of heart failure (HF) due to cardiac diseases with different etiologies (1). Among these changes, alterations in the amount and quality of the extracellular matrix components such as the collagen array, have been defined that lead in myocardial remodeling and finally impaired left ventricular (LV) function and promote the development of HF. A significant amount of fibrillar collagen depositions, which results in fibrosis of interstitial and perivascular region, has been reported in the cardiac ventricles of patients with arterial hypertension $(2,3)$. Imbalance between the production and degradation in extracellular collagen results in increase of collagen synthesis biomarkers in the circulation. One of these biomarkers is the carboxy-terminal propeptide of type I procollagen (PIP). It is secreted in a $1: 1$ ratio during the synthesis of type I collagen from procollagen type I (4). Serum PIP levels accurately mirror myocardial type I collagen synthesis (3). High level of serum PIP was found to be correlated with adverse consequences in hypertension and myocardial infarction $(5,6)$. The significance of PIP in the management of congestive heart failure

${ }^{1}$ Adana City Hospital, Health and Science University, Cardiology Department, Adana, Turkey, and ${ }^{2}$ Bozok University, the Faculty of Medicine, Cardiology Department, Yozgat, Turkey

Address for correspondence: M. Kaplan, MD, Adana City Hospital, Health and Science University, Cardiology Department, Adana, Turkey. Phone: +90.5324631285 with low ejection fraction has not been studied thoroughly. Here, we aimed to determine the role of PIP in the diagnosis of chronic $\mathrm{HF}$, its value in predicting mortality and morbidity due to $\mathrm{HF}$ and its relation with B-type natriuretic peptide and C-reactive protein.

\section{Material and methods}

\section{Subjects}

This prospective study was performed between September 2012 and October 2013. Informed consent of each subject and approval of the local ethical committee were obtained accordingly. 87 patients in the HF group with New York Heart Association functional class III or IV and 80 age- and gender-matched healthy subjects were enrolled into the study. The exclusion criteria were as follows: HF of hypertensive etiology, acute coronary syndrome within last six months, presence of renal failure (glomerular filtration rate $<60 \mathrm{ml} / \mathrm{min}$ ), hepatic failure (elevated transaminase levels three times more than the upper limit), presence of congenital heart disease, moderate or severe valvulopathy, moderate or severe pericardial effusion, previous hypertrophic cardiomyopathy, moderate or severe pulmonary hypertension, chronic obstructive pulmonary disease, connective tissue disorders. There was no evidence of active infection, inflammatory disease, cancer at the time of evaluation. Inclusion criteria for chronic HF group were a left ventricular EF of less than $40 \%$ measured by echocardiography. All patients were under a standardized optimal therapeutic medication. The etiology of chronic heart failure was idiopathic dilated cardiomyopathy in 65 patients $(74.7 \%)$ and ischemic heart disease in $22(25.3 \%)$. 
$421-424$

The following data were also obtained: age, gender, height, weight, presence of cardiovascular risk factors. Cardiovascular risk factors were determined according to the following criteria: hypertension $(>140 / 90 \mathrm{mmHg}$ or treatment with medication within the last six months), diabetes mellitus (fasting plasma glucose $\geq 126 \mathrm{mg} / \mathrm{dl}$ or current use of antihyperglycemic drugs), cigarette smoking (in the last 2 years). Duration from the time of diagnosis to time of the enrollment was recorded as disease duration. All the subjects included in the study were followed for one year. Within this time period, number of hospitalization longer than 24 hours due to exacerbation of chronic heart failure was defined as morbidity while death due to cardiac etiology was defined as mortality.

\section{Echocardiography and blood pressure measurement}

Standard echocardiographic examinations were performed by using an Acuson Sequoia C 256 echocardiography system (Acuson, Mountain View, CA, USA) with a 2.5-MHz transducer by the cardiologist blinded to the clinical data of the subjects in accordance with rules set by the American Society of Echocardiography (7). For the simplicity of the analyses, data related to left ventricle EF and left atrial (LA) diameter were used.

The blood pressure (BP) levels were measured from the right and left arms of the subjects in a sitting position by one trained observer blind to the study in the echocardiography laboratory. $\mathrm{BP}$ was measured twice in five minutes interval. The average of the four BP measurements was used for analysis.

\section{Biochemical analyses}

At the beginning of the study, peripheral venous blood samples of each subject were drawn and centrifuged within 5 min of sampling at $3000 \mathrm{rev} . \mathrm{min}$ for $10 \mathrm{~min}$ at $4{ }^{\circ} \mathrm{C}$. Native serum samples were then stored at $-80^{\circ} \mathrm{C}$ until use. Commercially available radioimmunoassay kits were used according to the manufacturer's instructions for the determination of circulating levels of PIP (Procollagen PICP RIA Kit, Orion Diagnostica, Turku, Finland). The intra-assay coefficient of variation for the measurement of PIP was $3 \%$. B-type natriuretic peptide (BNP) value of each subject at the beginning was measured for all samples using sandwich immunoassays (Biosite Triage, San Diego, CA, USA) accordingly. The hs-CRP level was assayed using the immunoturbidimetric method (Spectra East Laboratories, Rockleigh, NJ, USA). Serum hs-CRP $<5 \mathrm{mg} / \mathrm{L}$ was considered the cutoff level in our laboratory.

\section{Statistical analysis}

The SPSS for Windows 18.0 package program was used for statistical analyses. Continuous variables were expressed as mean $\pm \mathrm{SD}$ while categorical variables were shown in frequencies (\%). Normality of the variables was analysed by Kolmogorov-Smirnov test. Since all the variables were not distributed normally, the chi-square test was used to analyze categorical variables whereas Mann-Whitney U and Kruskal Wallis tests were used to analyze continuous variables. Spearman correlation analysis was used to establish the association between PIP and other continuous variables. A p value $<0.05$ was considered to be statistically significant. To find diagnostic cut-off value of PIP for differentiation of patient with HF from subjects with normal LVEF, a receiver operating characteristic (ROC) curve analysis was constructed, and the area under the curve (AUC) was reported, which is considered representative of the discriminitive ability of the variable cut-off. Sensitivity and specificity values for the best cut-off variables were determined using ROC curve analysis. The cut-off levels of PIP were calculated using MedCalc 9.2.0.1 (MedCalc Software, Mariakerke, Belgium).

\section{Results}

167 subjects were enrolled into this prospective study. HF group with average age of $64 \pm 9$ years while the control group with average age of $62 \pm 10$ years. Age and gender distribution over the groups were statistically similar $(\mathrm{p}=0.097$ and 0.265 respectively).Also height,weight and heart rate in the groups were statistically similar. $63 \%$ of HF group $(n=55)$ and $49 \%$ of the control group $(n=39)$ were overweight or obese $(p=0.060) .17$ $\%$ of subjects in HF group $(\mathrm{n}=15)$ and $15 \%$ of the control group $(\mathrm{n}=12)$ had hypertension $(\mathrm{p}=0.694)$.

Clinical data of the subjects are shown in Table 1. Average disease duration was $31 \pm 16$ months in HF group. $82 \%$ of the patients $(\mathrm{n}=71)$ in HF group were hospitalized at least once during one-year follow-up due to aggrevation of heart failure. Mortality rate in HF group was $35 \%$ for one-year follow-up.

Echocardiographic and biochemical measurement results are shown in Table 2.

Echocardiographically measured LVEF of HF and the control groups were $27 \pm 3 \%$ and $62 \pm 3 \%$, respectively $(p<0.001)$. There was a strong correlation between LVEF and BNP $(\mathrm{p}<0.001$ and

Tab. 1. Clinical data of the subjects.

\begin{tabular}{lccc}
\hline & $\begin{array}{c}\text { The control } \\
\text { group }(\mathrm{n}=80)\end{array}$ & $\begin{array}{c}\text { HF group } \\
(\mathrm{n}=87)\end{array}$ & $\mathrm{p}$ \\
\hline Age (year) & $62 \pm 10$ & $64 \pm 9$ & $\mathrm{p}=0.097$ \\
Female/Male (\%) & $17 / 63(21 / 79)$ & $25 / 62(29 / 71)$ & $\mathrm{p}=0.265$ \\
DM (\%) & $29(36)$ & $31(36)$ & $\mathrm{p}=0.934$ \\
HT (\%) & $30(38)$ & $41(47)$ & $\mathrm{p}=0.209$ \\
Cigarette smoking (\%) & $31(39)$ & $42(48)$ & $\mathrm{p}=0.215$ \\
BMI (kg/m2) & $25.2 \pm 3.4$ & $26.0 \pm 3.8$ & $\mathrm{p}=0.171$ \\
SBP (mmHg) & $124 \pm 14$ & $121 \pm 15$ & $\mathrm{p}=0.181$ \\
DBP (mmHg) & $73 \pm 11$ & $73 \pm 12$ & $\mathrm{p}=0.533$ \\
Pulse Pressure (mmHg) & $50 \pm 7$ & $49 \pm 7$ & $\mathrm{p}=0.183$ \\
\hline
\end{tabular}

DM - Diabetes Mellitus, HT - Hypertension, BMI - Body Mass Index, SBP - Systolic Blood Pressure, DBP - Diastolic Blood Pressure

Tab. 2. Echocardiographic and biochemical measurement results of the subjects.

\begin{tabular}{lccc}
\hline & $\begin{array}{c}\text { The control } \\
\text { group }(\mathrm{n}=80)\end{array}$ & $\begin{array}{c}\text { HF group } \\
(\mathrm{n}=87)\end{array}$ & $\mathrm{p}$ \\
\hline LVEF $(\%)$ & $62 \pm 3$ & $27 \pm 3$ & $\mathrm{p}=0.000$ \\
LA diameter $(\mathrm{mm})$ & $36 \pm 2$ & $47 \pm 5$ & $\mathrm{p}=0.000$ \\
hs-CRP $(\mathrm{mg} / \mathrm{dl})$ & $1.08 \pm 0.46$ & $4.03 \pm 2.12$ & $\mathrm{p}=0.000$ \\
BNP $(\mathrm{pg} / \mathrm{ml})$ & $80 \pm 40$ & $2094 \pm 883$ & $\mathrm{p}=0.000$ \\
PIP $(\mu \mathrm{g} / \mathrm{L})$ & $86 \pm 16$ & $137 \pm 15$ & $\mathrm{p}=0.000$ \\
\hline
\end{tabular}

LVEF - Left Ventricle Ejection Fraction, LA - Left Atrium, hs-CRP - High Sensitive C-reactive Protein, BNP - B type natriuretic peptide, PIP - The Carboxy-terminal Propeptide of Type I Procollagen 


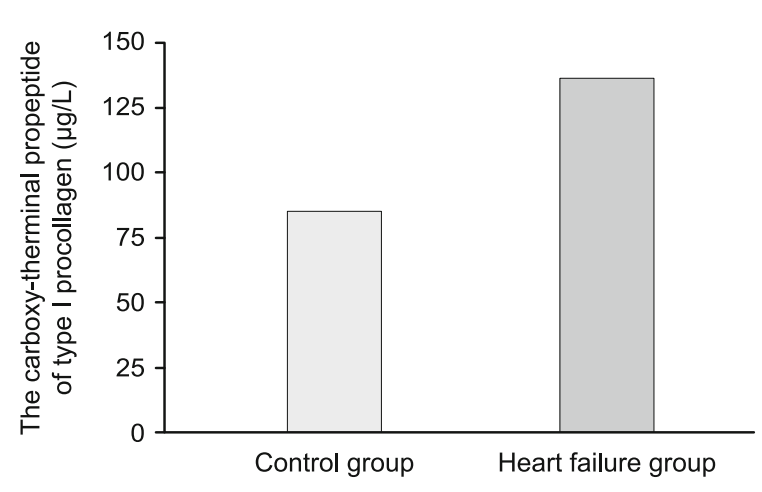

Fig. 1. The comparison of groups in respect to the carboxy-terminal propeptide of type I procollagen.

$\mathrm{r}=-0.828)$. Similarly, LA diameter of $\mathrm{HF}$ and the control groups were $36 \pm 2 \mathrm{~mm}$ and $47 \pm 5 \mathrm{~mm}$ respectively $(\mathrm{p}<0.001)$ and LA diameter revealed significant correlation with BNP values $(\mathrm{p}<$ 0.001 and $r=0.781$ ). Average BNP and hs-CRP values of HF group were significantly higher compared to those of the control group $(\mathrm{p}<0.001)$. Average PIP value of HF group was significantly higher than that of the control group $(\mathrm{p}<0.001)$ (Fig. 1). Both hs-CRP and BNP values were well correlated to PIP values ( $\mathrm{p}<$ $0.001, r=0.620$ and 0.728 respectively). Among HF group, PIP value of patients who died at the end of one year was similar to that of patients survived at the end of first year $(p=0.718)$. Similarly, there was not a significant correlation between the number of hospitalization and PIP values among patients in HF group ( $p$ $=0.713 \mathrm{r}=-0.040)$. Patients with DM $(\mathrm{n}=60)$ had similar PIP values compared to non-diabetics $(n=107)(p=0.579)$. Similarly, hypertensive patients $(\mathrm{n}=27)$ didn't differ from the patients with normotensive inviduals $(n=140)(\mathrm{p}=0.257)$.

Optimal threshold cut-off point for PIP to differentiate subjects with normal LVEF from patients with HF was calculated by ROC analysis. PIP value of $119 \mu \mathrm{g} / \mathrm{L}$ was found to be cut-off value with $86.2 \%$ sensitivity and $98.8 \%$ spesificity to detect $\mathrm{HF}$ (AUC: 0.984 with $95 \%$ CI: 0.951 to 0.997 and $p$ value $<0.001$ ). If PIP value of $>119 \mu \mathrm{g} / \mathrm{L}$ was accepted as abnormal, frequency of abnormal PIP in the control and HF groups were $5 \%$ and $87 \%$ respectively $(\mathrm{p}<0.001)$.

\section{Discussion}

Chronic heart failure is one of the leading causes of cardiovascular morbidity and mortality. Usually it appears as an end stage of cardiovascular diseases such as coronary artery disease, hypertension, or valvulopathies. Although echocardiography is one of the best tools for diagnosis and managment of heart failure, it may not be sufficient enough to reflect clinical status or reponse to medication. For these purposes, there are biomarkers introduced to estimate clinical status such as BNP, the amino-terminal fragment of pro-B-type natriuretic peptide (NT-proBNP), and hsCRP. Herein, we aimed to search the role of PIP in diagnosis and follow-up of heart failure.
Löfsjögård et al showed that increased collagen synthesis measured by PIP was independently correlated to decreased LVEF and increased BNP levels (8). But their study population included older patients with higher SBP and relatively higher LVEF without control group for comparison. Our study included patients (n $=167)$ with average age of $63 \pm 9$ years and average SBP of 122 $\pm 15 \mathrm{mmHg}$. In our study, we also found that serum PIP level (n $=167)$ was well correlated to LVEF $(r=-0.745, p<0.001)$ and BNP levels $(r=0.728, p<0.001)$. In the literature, there have been studies concerning PIP in different clinical states such as hypertension, acute myocardial infarction, heart failure, and diabetics but they didn't adress a cut-off value to differentiate heart failure with low ejection fraction from otherwise normal patients $(8,9)$. In our study, we performed ROC analysis to obtain cut-off value. PIP value of $119 \mu \mathrm{g} / \mathrm{L}$ was found to be cut-off value with $86.2 \%$ sensitivity and $98.8 \%$ spesificity to detect HF with low ejection fraction (AUC:0.984 with 95\% CI: 0.951 to 0.997 and $\mathrm{p}<0.001$ ). There are cut-off values for BNP to differentiate low-ejectionfraction heart failure.PIP can improve diagnostic accuracy of BNP in these states since it reflects structural myocardial involvement rather than pressure or volume changes.

BNP or NT-proBNP have been extensively studied in managment of heart failure both with normal and low ejection fraction $(10,11)$. They are found to increase proportionally with degree of heart failure. But clinical aspect of this finding is controversial since studies related to natriuretic peptide-guided versus symptomguided therapy showed conflicting results (12). Major reason for such result may be due to complex nature of heart failure in which medical response can not be explained by a simple molecule. In our study, we also found that average BNP value was significantly higher in HF group compared to control group $(2094 \pm 883 \mathrm{pg} / \mathrm{ml}$ vs. $80 \pm 40 \mathrm{pg} / \mathrm{ml}, \mathrm{p}<0.001)$. Also, BNP of patients with mortality at the end of one-year follow-up was significantly higher than HF patients survived beyond one year $(2564 \pm 964 \mathrm{pg} / \mathrm{ml}$ vs. $1846 \pm$ $732 \mathrm{pg} / \mathrm{ml} \mathrm{p}<0.005)$. Similarly, among patients with HF, patients hospitalized at least once in a year $(n=71)$ had statistically higher BNP value than patients without any morbidity $(\mathrm{n}=16)(2233$ $\pm 889 \mathrm{pg} / \mathrm{ml}$ vs. $1477 \pm 536 \mathrm{pg} / \mathrm{ml}, \mathrm{p}<0.005)$. Although strong correlation of BNP with mortality and morbidity was found, we couldn't demonstrate similar type of relation with PIP. Among patients with HF, PIP did not show significant relation with either mortality $(\mathrm{p}=0.718)$ or morbidity (at least one time hospitalization due to HF) $(p=0.352)$. These results are not contadicting the literature because BNP is predominately released from the left and the right cardiac ventricle in regards to volume and pressure overload (13). However, PIP level is directly related with level of myocardial fibrosis and it reflects collagen turnover in the myocardium. Thus, myocardial injury rather than volume or pressure overload is important in release of PIP into the circulation. Kop et al found that microinjury detected by Troponin $\mathrm{T}$ was well correlated with PIP in patients with HF (14). Our study population consisted of relatively stable patients in respect to myocardial fibrosis since we excluded acute coronary syndrome at the time of enrollement. Also we didn't carry out serial measurements at the time of each hospitalization. Cerisano et al found that patients with 
acute myocardial infarction revealed persistant increment of PIP from day 1 to month 6 , reflecting the healing process of injured myocardium (15). Thus, new surge in the stationary level of increased PIP necessitates new cardiac events ending with fibrosis. As a result, our findings concerning mortality and morbidity do not contradict the findings in the literature.

Increased hs-CRP was found to be well correlated to increased collagen turnover which is measured by PIP (16). In other words, cardiac fibrosis is an inflammatory process and hs-CRP promotes structural remodelling of the heart (17). We also found that patients with HF had significantly higher hs-CRP $(p<0.001)$. Additionally, PIP was increasing proportionally with hs-CRP levels among all subjects $(\mathrm{p}<0.001, \mathrm{r}=0.620)$.

In conclusion, heart failure with low LVEF is an end result of cardiac remodelling along with fibrosis which can be measured with PIP. Patients with HF had significantly higher level of PIP compared to otherwise normal subjects. PIP is a molecule reflecting structural alterations in the heart. Thus, it may not mirror acute events in follow-up of chronic heart failure but it is very beneficial biomarker in diagnosis of low-LVEF heart failure with high sensitivity and specificity.

\section{References}

1. López B,16 González A, Díez J. Circulating Biomarkers of Collagen Metabolism in Cardiac Diseases. Circulation 2010; 121: 1645-54.

2. Weber KT. Fibrosis and hypertensive heart disease. Curr Opin Cardiol 2000; 15: 264-272.

3. Querejeta R, López B, González A et al. Increased collagen type I synthesis in patients with heart failure of hypertensive origin: relation to myocardial fibrosis. Circulation 2004; 110: 1263-1268.

4. González A, López B, Ravassa S et al. Biochemical markers of myocardial remodelling in hypertensive heart disease. Cardiovasc Res 2009; 81: 509-518.

5. Poulsen SH, Host NB, Egstrup K. Long-term changes in collagen formation expressed by serum carboxyterminal propeptide of type-I procollagen and relation to left ventricular function after acute myocardial infarction. Cardiology 2001; 96: 45-50.

6. Zannad F, Alla F, Dousset B, Perez A, Pitt B. Limitation of excessive extracellular matrix turnover may contribute to survival benefit of spironolactone therapy in patients with congestive heart failure: insights from the Randomized Aldactone Evaluation Study (RALES) Circulation 2000; 102: 2700-2706.

7. Lang RM, Bierig M, Devereux RB, Flachskampf FA, Foster E, Pellikka PA et al. Chamber Quantification Writing Group, American Society of Echocardiography's Guidelines and Standards Committee, European
Association of Echocardiography.Recommendations for chamber quantification: a report from the American Society of Echocardiography's Guidelines and StandardsCommittee and the Chamber Quantification Writing Group, developed in conjunction with the European Association of Echocardiography, a branch of the European Society of Cardiology. J Am Soc Echocardiogr 2005; 18: 1440-1463.

8. Löfsjögård J, Kahan T, Díez J, López B, González A, Edner M, Henriksson P, Mejhert M, Persson H. Biomarkers of collagen type I metabolism are related to B-type natriuretic peptide, left ventricular size, and diastolic function in heart failure. J Cardiovasc Med (Hagerstown). 2014; 15: 463-469.

9. Jellis C, Wright J, Kennedy D, Sacre J, Jenkins C, Haluska B, Martin J, Fenwick J, Marwick TH. Association of imaging markers of myocardial fibrosis with metabolic and functional disturbances in early diabetic cardiomyopathy. Circ Cardiovasc Imaging. 2011; 4: 693-702.

10. Sun RR, Lu L, Liu M, Cao Y, Li XC, Liu H, Wang J, Zhang PY. Biomarkers and heart disease. Eur Rev Med Pharmacol Sci. 2014; 18 : 2927-2935.

11. Tate S, Griem A, Durbin-Johnson B, Watt C, Schaefer S. Marked elevation of B-type natriuretic peptide in patients with heart failure and preserved ejection fraction. J Biomed Res 2014; 28: 255-261.

12. De Vecchis R, Esposito C, Di Biase G, Ariano C, Giasi A, Cioppa C. B-type natriuretic peptide-guided versus symptom-guided therapy in outpatients with chronic heart failure: a systematic review with meta-analysis. J Cardiovasc Med (Hagerstown). 2014; 15: 122-134.

13. Mueller C, Breidthardt T, Laule-Kilian K, Christ M, Perruchoud AP. The integration of BNP and NT-proBNP into clinical medicine. Swiss Med Wkly. 2007; 137: 4-12.

14. Kop WJ, Gottdiener JS, deFilippi CR, Barasch E, Seliger SL, Jenny NS, Christenson RH. Cardiac microinjury measured by troponin T predicts collagen metabolism in adults aged $>=65$ years with heart failure. Circ Heart Fail. 2012; 5: 406-413.

15. Cerisano G, Parodi G, Dovellini EV, Migliorini A, Tommasi M, Raspanti S, Buonamici P, Taddeucci E, Valenti R, Antoniucci D. Time course of serum collagen types I and III metabolism products after reperfused acute myocardial infarction in patients with and without systemic hypertension. J Hum Hypertens. 2009; 23: 40-47.

16. Zhang R, Zhang YY, Huang XR, Wu Y, Chung AC, Wu EX, Szalai AJ, Wong BC, Lau CP, Lan HY. C-reactive protein promotes cardiac fibrosis and inflammation in angiotensin II-induced hypertensive cardiac disease. Hypertension. 2010; 55: 953-960.

17. Sasaki N, Okumura Y, Watanabe I, Mano H, Nagashima K, Sonoda K, Kogawa R, Ohkubo K, Nakai T, Hirayama A. Increased levels of inflammatory and extracellular matrix turnover biomarkers persist despite reverse atrial structural remodeling during the first year after atrial fibrillation ablation. J Interv Card Electrophysiol 2014; 39: 241-249.

Received February 26, 2018. Accepted April 5, 2018. 